Historic, Archive Document

Do not assume content reflects current scientific knowledge, policies, or practices. 

Bulletin No. 3

SPRING, 1915

March 1, 1915

\section{Trade List of Nursery Stock OFFERED FOR SALE AT}

\section{W I L I S' NURSERIES}

A. WILLIS \& CO., Proprietors. OTTAWA, KANSAS.

\section{$1 \mathrm{~kg}$}

OTTAWA, KANSAS.
The following is all thrifty and well grown No. 1 stock of the slzes and ages named. Packing will be done at cost, but no charge will we made for drayage to the depot or express office. For stock gold terms are cash or satisfactory security before shipment. ENTOMOLOGIST'S CERTIFICATE WITH EVERY SHIPMENT.

APPLES.

Strictly Choice Trees.

11-16 inch and up.

$5 / 8$ to $11-16$ inch

$1 / 2$ to $5 / 8$ inch

3 to 4 feet branched

2 to 3 feet

Tarieties starred 1c per tree less in 11-16 srade. Delicious 2c per tree extra in each grade.

*Arkansas Black.. 800

Atm. Strawberry.. 25

Bayard .............. 30

Ben Davis ............300

Bismarck ..............

Clayton ............. 200

Delicious.....$\cdots \cdots$

Dominie ............ 175

Early Harvest .... 100

Fall Pippin .......200

Fameuse ........... T5

* Gano .............1000

Gilpin .............. 100

Grimes Golden .... 300

Tungram ............. 75

Janet $\ldots$......................

Jonathan ...........4500 35

*Lansingburg ...... 400

Lowell $\ldots \ldots \ldots \ldots \ldots . .20$

Maiden's Blush..... 275

Mam Black Twig.. 375

Mammoth Pippin.. 90

*Mann ............ 200

Tilam

Minkler............. 100

Northern Spy ....... 10

Newtown Pippin... 500

N. W. Greening.... 275

opalescent ............

Red Astrachan........

Fied June ............

Fiambo ............. 25

Roman Stem .......

Rome Beauty ...... 125

Salome ............. 16

*Smith's Cider ....4 400

$\checkmark-$ Saps of Wine...... 12

*Spitzenberg. ......5 500

Stayman Winesap 4500

Sweet June ....... 100

Tallman Sweet.... 250

*Vagener

Walbridge ............. 125

Winesap ........... 975

Winter Banana....66 650

Wismer's Desert... 400

W. W. Pearmain... 250

Wolf River

Yel. Bellefiower..... 60

Yel. Horse.............

Yel. Transparent.

*York Imperial ....3400

$\ddot{125}$

44
CRABS.

Each

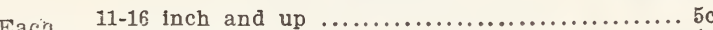

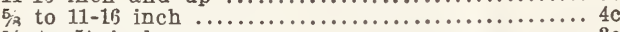

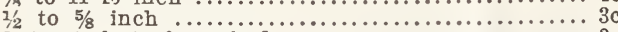

3 to 4 feet, branched ......................

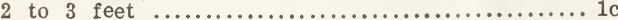

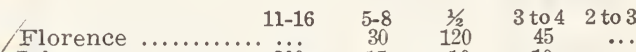

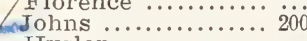

Hyslop

$\begin{array}{rrrr}30 & 120 & 45 & \ldots \\ 15 & 10 & 10 & \ldots \\ 20 & 50 & \ldots & \ldots \\ 40 & 40 & 12 & \ldots \\ 30 & 25 & 20 & \ldots \\ 100 & 100 & 20 & 59 \\ 50 & \because \ddot{2} & 60 & 35 \\ 30 & 12 & 7 & \ldots\end{array}$

Martha

Quaker Beauty .... 175

Transcendant ...... 400

Whitney $\ldots \ldots \ldots \ldots$
Yel. Siberian $\ldots \ldots \ldots$
25

STANDARD PEARS.

Each

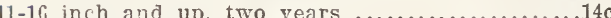

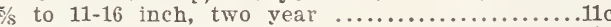

$1 / 2$ to $5 / 8$ inch, two year .....................

$s / 8$ to $1 / 2$ inch, two year ....................... $5 c$

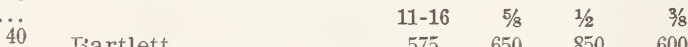

................ $575 \quad 650 \quad 850 \quad 600$

P. d'Anjou .............200 $200 \quad 275 \quad 175 \quad 60$

Clapp's Favorite ........250 250 $175 \quad 100 \quad 220$

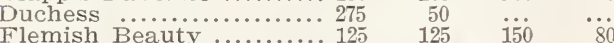

Garber ................... $300 \quad 100 \quad 50$

Howell .................. $100 \quad 75 \quad 125$

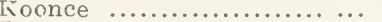

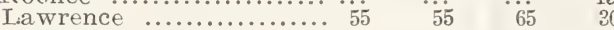

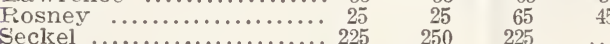

Sheldon

Vernon Beauty ............ 45

KEIFFER PEARS.

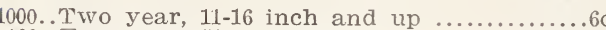

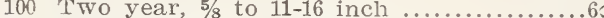

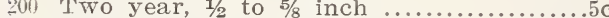

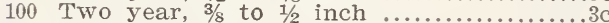

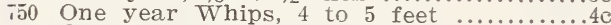

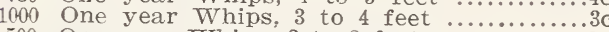

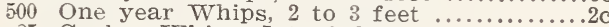

25 Garber Whips, 5 to 6 feet ................

DWARF PEARS.

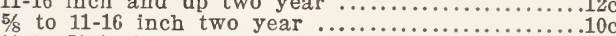

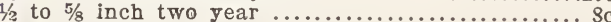

$3 / 8$ to $1 / 2$ inch two year ......................4c

Bartlett

$11-1$

$5 / 8 \quad 1 / 2$

$3 / 8$

24 B. d'Anjou ................ 30

Clapp's Favorite .......... T5

Duchess

Duchess

Keiffer

Louise $\mathrm{Bon}$

Seckel 


\section{CHERRILS.}

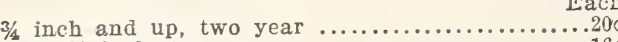

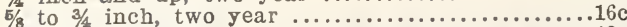

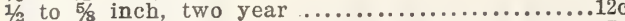

$3 / 3$ to $1 / 2$ inch, two year .....................

$\begin{array}{lllll}3 / 4 & 5 / 8 & 1 / 2 & 3 / 8\end{array}$

Bing

Black Tärtarian ...............

Dyehouse

$\begin{array}{lll}50 & \cdots & \cdots \\ 30 & \ddot{2} 0 & 20\end{array}$

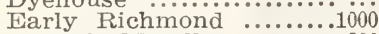

English Morello ......... 300

Louis Phillip .............. 200

Lambert ................... 100

May Duke ................... 25

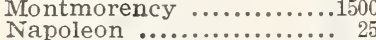

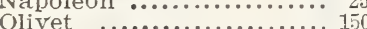

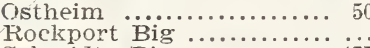

Schmidts Big ............ 17

Winsdor ................ 15

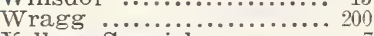

Ýllow Spanish

CHERRIES-One Year.

2000 Montmorency, 11-16 inch and up.

PLCM ON PIUM.

$8 / 4$ inch and up, two year

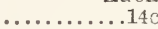

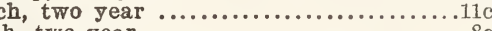

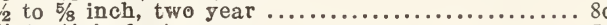

to $1 / 8$ inch, two year

Abundance $\quad \frac{3 / 4}{5 / 8} \quad \frac{1 / 2}{3 / 8}$

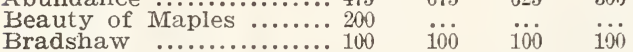

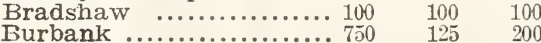

Coes Golden

Climax ....................

German Prune .............. 800

Genii Green Gage................. 120

Imperial Gage .............. 20

Lombard ..................250 175

$\ddot{125} \quad \ddot{175}$

$75 \quad 100$

Monarch

Moore's Arctic .............. 25

Fond's Seedling .......... 55

Fied June ....................

Reene Claude ............. 100

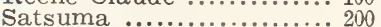

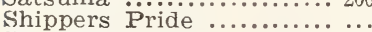

Shrop. Damson ............ 100

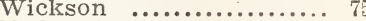

Yellow Egg .........................

\section{PLUM ON PEACH.}

11-16 inch and up ..............................

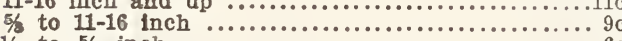

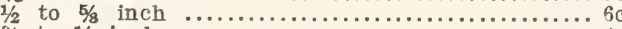

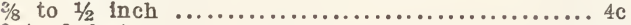

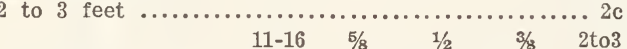

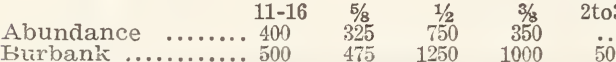

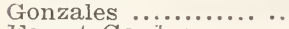

Forest Garden...........

Mariana

$1250 \quad 1000$

18

ced June

Weaver $\ldots \ldots \ldots \ldots \ldots \ldots \ldots$

Wickson

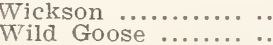

APRICOTS

Very Fine Trees, one and two years

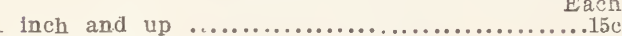

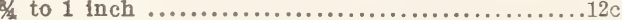

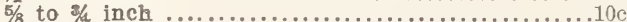

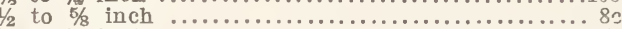

$8 / 8$ to $1 / 2$ inch

2 to 3 feet

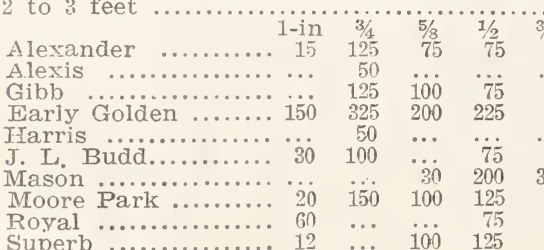

QUINCE.

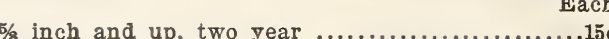

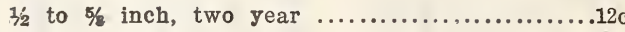

$3 / 8$ to $1 / 2$ inch, two year ...................... $8 \mathrm{c}$

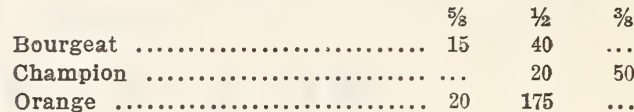

\section{NECTARINES.}

75 Nectarines $5 / 8$ inch and $u$.

75) Boston 9-16 inch and up ..................10

50 Boston, $7-16$ to $9-16$ inch ............... 7c

275 Boston, 5-16 to $7-16$ inch ................ 5c

200 Boston, 2 to 3 feet ...................... 3c

\section{PEACH.}

$5 / 8$ inch and up

Each

$9-16$ inch and up $\ldots \ldots \ldots \ldots \ldots \ldots \ldots \ldots \ldots \ldots . . .11 / 2 c$

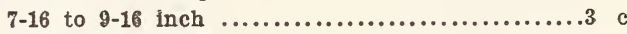

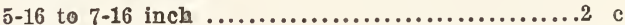

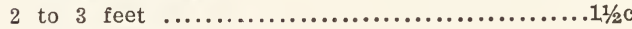

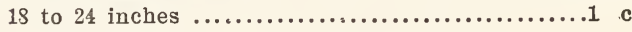

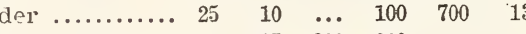

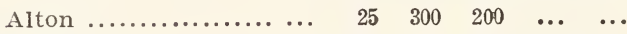

$\begin{array}{llllllll}\text { Amsden } & . . . . . . . . . . .6 & 25 & \ldots & \ldots & 30 & 70 & \ldots\end{array}$

$\begin{array}{lllllllll} & \text { Ark. Traeler } \ldots . . . . .2 & 25 & \ldots & \ldots & 50 & 60 & \ldots\end{array}$

$\begin{array}{rrrrrrr}\text { Belle of Georgia........ } & 30 & \ldots & \ldots & \ldots & \ldots \\ \text { Blood Cling ......... } & \ldots & \ldots & 50 & 75 & 40 & 32\end{array}$

$\begin{array}{llllllll}\text { Blood Free } & . . . . . . . . . & 10 & \ldots & 75 & 100 & 85 & \ldots\end{array}$

$\begin{array}{lllllll}\text { Bokara .............. } 25 & 75 & 200 & 500 & 400 & \ldots\end{array}$

$\begin{array}{llllllll}\text { Carman ............. } 25 & 100 & 100 & 150 & \ldots & \ldots\end{array}$

Champion ........... $30 \quad 200 \quad 1500 \quad 2250 \quad 750 \quad 150$

Chinese Cling .......... $30 \quad 30 \quad 75 \quad \ldots . . . \quad \ldots$

Crawford's Early....... $\quad \begin{array}{llllll}75 & 1000 & 1275 & 1200 & 335\end{array}$

Crawford's Late .... ... $\quad 75 \quad 750 \quad 1025 \quad 1000 \quad 950$

$\begin{array}{lllllll}\text { Crosby } \ldots \ldots \ldots \ldots \ldots \ldots & 25 & 200 & 1400 & 950 & 39\end{array}$

$\begin{array}{lllllll}\text { Early Rivers ........ } & 75 & 75 & 20 & 50 & 30 & \ldots\end{array}$

Early York ........... $200 \quad 300 \quad 100 \quad 30 \quad \ldots$

Elberta ............ $500 \quad 5000 \quad 7500 \quad 9000 \quad 9000 \quad 1750$

Family Favorite .... $20 \quad 14 \quad 70 \quad 30 \quad 25 \quad \ldots$

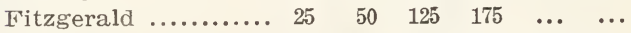

Foster .............. 25 $50 \quad 200 \quad 250 \quad 200 \quad \ldots$

F'ox seedling ........ 50 ... $50300 \quad 150 \quad 50 \quad \ldots$

Globe ................. $75 \quad 500 \quad 350 \quad 250 \quad \ldots$

$\begin{array}{llllllllll}\text { Greensboro } & \ldots . . . \ldots \ldots & \ldots & 100 & \ldots & \ldots & \ldots & \ldots\end{array}$

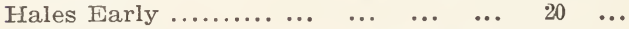

$\begin{array}{llllllll}\text { Heath Cling ........ } 25 & 50 & 250 & 275 & 750 & \ldots\end{array}$

Indian Cling . .......... $\quad 75 \quad 175 \quad 300 \quad 200 \quad 50$

Japan Blood Dwarf.. ... $\quad 50 \quad 250 \quad 250 \quad 175 \quad$...

I.emon Cling ........... $100 \quad 700 \quad 500 \quad 425 \quad 8$

$\begin{array}{llllllll}\text { Mamie Ross ......... } & 50 & \ldots & \ldots & \ldots & 70 & \ldots\end{array}$

$\begin{array}{lllllll}\text { Mratthew's Beauty .. } & 10 & \ldots & 250 & 200 & 140 & \ldots\end{array}$

$\begin{array}{llllllll}\text { Miayflower ........... } 20 & 25 & 25 & \ldots & \ldots & 45\end{array}$

$\begin{array}{lllllllll}\text { Mountain Rose } & \ldots . . & 10 & \ldots & 20 & 75 & \ldots & \ldots\end{array}$

Niagara ............... $20 \quad 275 \quad 275 \quad 250 \quad \ldots$

Old Mixon Cling........ $\quad \begin{array}{llllll}75 & 225 & 200 & 75 & \ldots\end{array}$

$\begin{array}{lllllll}\text { Old Mixon Free ..... } & 125 & \ldots & 25 & \ldots & 50 & \ldots\end{array}$

Salway .............. $25 \quad 200 \quad 400 \quad 500 \quad 250 \quad \ldots$

$\begin{array}{lllllll}\text { smock ............... } 50 & 125 & 400 & 450 & 240 & \ldots\end{array}$

Sneed .................. $75 \quad 375 \quad 400 \quad 240 \quad \ldots$.

Stinson's October.... $20 \quad 10 \quad 100 \quad 65 \quad \ldots$.

$\begin{array}{llllllll}300 & 25 & 25 & 500 & 600 & 275 & \ldots\end{array}$

Triumph ............ $75 \quad 75 \quad 25 \quad 30 \quad 575$

Wonderful ........... $20 \quad 50 \quad 225 \quad 500 \quad 475$ 


\section{GRAPES.}

Per M

100 Agawam, 1 year, No. 1 ............. $\$ 30.00$

400 Brighton, 1 year, No. 1 ............. 30.00

12000 Concord, 2 year, No. 1 .............. 18.00

18000 Concord, 1 year, No. 1 ............. 14.00

300 Campbells Eariy, 2 year, No. 1 ...... 70.00

500 Campbells Early, 1 year, No. 1 ..... 50.00

100 Delaware, 1 year, No, 1 ............ 30.00

50 Dracut Amber, 1 year, No. 1 ........ 30.00

200 Ive's Seedling, 2 year, No. 1 ........ 30.00

750 Moore's Diamond, 1 year, No. 1 ..... 25.00

1800 Moore's Early, 2 year, No. 1 ....... 40.00

4500 Moore's Early, 1 year, No. 1 ........ 30.00

400 Moore's Early, 1 year, No. 2 .......... 20.00

900 Niagara, 2 year, No. 1 ............. 35.00

4000 Niagara, 1 year, No. 1 ............. 25.00

600 Niagara, 1 year, No. 2 .............. 15.00

750 Pockington, 1 year, No. 1 ............ 25.00

150 Pockington, 1 year, No. 2 ........... 15.00

2500 Worden, 2 year, No. 1 .............. 30.00

1200 Warden, 1 year. No. 1 .............. 20.00

600 Warden, 1 year, No. 2 .............. 12.50

\section{GOOSHBHRRIES.}

1500 Downing 2 Each

1200 Houghton, 2 year, No. 1 ................. 3 c

150 Pearl, 2 year, No. $1 \ldots \ldots \ldots \ldots \ldots \ldots \ldots .61 \frac{1}{2} \mathrm{c}$

150 Smith's Improved, 2 year, No. $1 . \ldots \ldots . .66$ c

\section{CUFRANTS.}

200 Black Champion, 2 year

200 Cherry, 2 year, No. 1 ................. c

250 Fays Prolific, 2 year No. $1 \ldots \ldots \ldots \ldots . . . .3$ c

150 Knights Late Red, 2 year, No. $1 \ldots \ldots \ldots . . .3$

200 Red Dutch, 2 year, No. 1 .................2 $1 / 2 \mathrm{c}$

100 White Grape, 2 year, No. 1 .....................

\section{RASPBERRIES.}

3000 Cardinal .................................

500 Kansas ................................ 6.00

\section{BLACKBERRIES.}

1000 Early Harvest

Per M.

(1).............. $\$ 10.00$

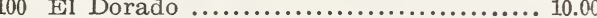

500 Mercereau .................................. 12.00

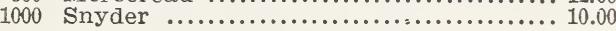

\section{STRAWBWRRIES.}

General Assortment

Per M.

August Luther

Bederwood

Brandywine

Charles I

Captain Jack

Clyde

Crescent

Excelsior

Gandy

Glen Mary
Haveland
Jesse
Michael's Early
Robinson
Sample
Semator Dunlap
Splendid
Harfield

\section{IULBERRIES.}

15 Downing, 2 to 3 feet $\ldots . . . .$.

15 New American, 3 to 4 feet .............. 7c

20 New American, 2 to 3 feet ............... 5c

25 Russian, $11 / 2$ inches caliper ...............15c

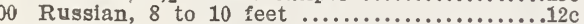

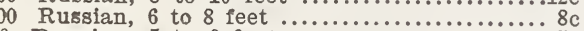

50 Russian, 5 to 6 feet .....................

100 Russian, 4 to 5 feet ...................4c

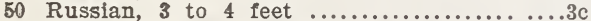

For Russian Mulberry Seedlings see Forest Seedlings.

\section{ASPARAGUS.}

Per M.

10000 Conover's Colossal, 2 year, No. $1 \ldots \ldots \ldots . \$ 3.00$

10000 Giant Argenteuil, 2 year, No. $1 \ldots \ldots \ldots . . .30$

\section{MISCELLANEOUS.}

Per M.

1500 Dewberries, Austin ................ 8.00

500 Dewberries, Leucretia ............ 8.00

500 Himalaya Berries .................. 25.00

200 Juneberries, 2 to 3 feet ............. 40.00

2000 Horse Radish ...................... 6.00

5000 Rhubarb, 1 year; No. 1 .............. 10.00

\section{ORNAMENTAL TREES.}

Each

4 Ash, 2 inch caliper ......................25c

15 Ash, $1 \frac{1 / 2}{2}$ to 2 inch caliper .................20

40 Ash, 6 to 8 feet ....................... 8 8

30 Balm of Gilead, 8 to 10 feet ............... $12 \mathrm{c}$

75 Balm of Gilead, $\epsilon$ to 8 feet ............... 9c

50 Balm of Gilead, 5 to 6 feet .............. 7c

25 Balm of Gilead, 3 to 5 feet .............. 5c

25 Box Elder, 8 to 10 feet....................14

300 Box Elder, 6 to 8 feet...................

250 Box Elder, 5 to 6 feet.................. ic

325 Box Elder, 4 to 5 feet.................. $4 \mathrm{c}$

500 Box Elder, 3 to 4 feet.................. 2c

100 Catalpa Bungeii, 6 foot stems .............490

325 Catalpa Bungeii, 5 to 6 foot stems.........30c

150 Catalpa Bungeil, 4 to 5 foot stems.......25c

50 Catalpa Speciosa, 6 to 8 feet ............10

25 Catalpa Speciosa, 5 to 6 feet ........... 8c

25 Catalpa Speciosa, 4 to 5 feet ............ 5c

5 Chestnut Am Sweet, 5 to 6 feet ..........15c

925 Elm, 6 to 8 feet ......................

$850 \mathrm{Elm}, 5$ to 6 feet $\ldots \ldots \ldots \ldots \ldots \ldots \ldots \ldots \ldots \ldots \ldots \ldots \ldots \ldots$

275 Elm, 4 to 5 feet ..................... $5 \mathrm{c}$

20 Linden European, 4 to 5 feet .............15c

30 Locust, Black, $2 \frac{1}{2}$ inch caliper ..................

50 Locust, Black, 2 to $2 \frac{1}{2}$ inch caliper.......20 c

150 Locust, Black, $1 \frac{1}{2}$ to 2 inch caliper........15c

525 Locust, Black, 8 to 10 feet .................12

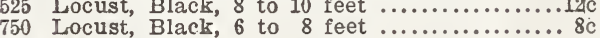

750 Locust, Black, 6 to 8 feet $\ldots \ldots \ldots \ldots \ldots \ldots \ldots \ldots \ldots .6 \%$

800 Locust, Black, 4 to 5 feet $\ldots \ldots \ldots \ldots \ldots \ldots, 4 \mathrm{c}$

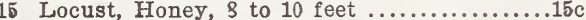

15 Locust, Honey, 8 to 10 feet $\ldots \ldots \cdots \cdots \cdots \cdots \cdots \cdots \cdots \cdots \cdots \cdots \cdots \cdots \cdots \cdots \cdots$

275 Locust, Honey, 6 to 8 feet $\ldots \ldots \cdots \cdots \cdots \cdots \cdots \cdots \cdots \cdots \cdots \cdots \cdots \cdots \cdots$

275 Lacust, Honey, 5 to 6 feet $\ldots \ldots \ldots \ldots \cdots \cdots \cdots \cdots \cdots \cdots$

175 Locust, Honey, 3 to 4 feet ..................

10 Maple Norway, 8 to 10 'feet ..............35c

25 Maple, Soft, $11 / 2$ to 2 inch caliper..............15c

925 Maple, Soft, $11 / 2$ to 2 inch caliper ...........15c

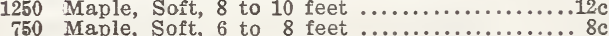

750
575
$\mathbf{5 a p l e}$

650 Maple, Soft, 4 to 5 feet $\ldots \ldots \ldots \ldots \ldots \ldots \ldots \ldots$ tc

8 Mountain Ash, 5 to 6 feet ..............10

12 Mountain Ash, 4 to 5 feet ................... $8 \mathrm{c}$

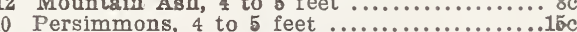

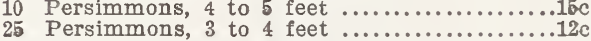

20 Poplar, Carolina, 2 inch caliper................25

25 Poplar, Carolina, $1 \frac{1 / 2}{20} 2$ inch caliper ......12

875 Poplar, Carolina, 8 to 10 feet ............. sc

12 Poplar, Lombardy, 8 to 10 feet ....................

12 Poplar, Lombardy, 8 to 10 feet $\ldots \ldots \ldots \ldots \ldots \ldots \ldots \ldots \ldots . .12 \mathrm{c}$

200 Poplar, Norway, 8 to 10 feet ..................

875 Poplar, Norway, 6 to 8 feet .............6c

175 Poplar, Norway, 5 to 6 feet $\ldots . . . . . . . . . . .4 c$

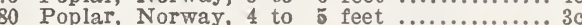

20 Poplar, Silver, 8 to 10 feet .................15

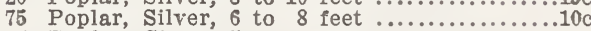

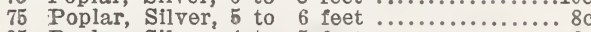

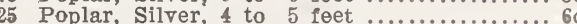

25 Poplar, Silver, 3 to 4 feet $\ldots \ldots \ldots \ldots \ldots \ldots \ldots \ldots \ldots \ldots \ldots \ldots$ c

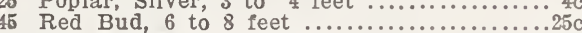

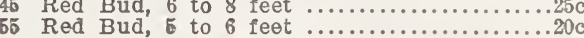

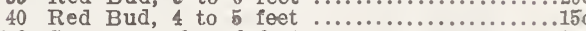

500 Sycamore, 6 to 8 feet $\ldots \ldots \ldots \ldots \ldots \ldots \ldots \ldots \ldots \ldots$

750 Sycamore, 5 to 6 feet $\ldots \ldots \ldots \ldots \ldots \ldots \ldots \ldots . .6 \mathrm{c}$

375 Sy amore, 4 to 5 feet $\ldots \ldots \ldots \ldots \ldots \ldots \ldots \ldots \ldots, \ldots$

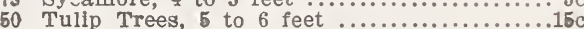

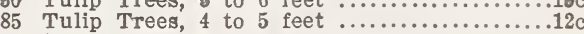


Each

T5 Almond, Pink, 2 to 3 feet ..................

120 Almond, Pink, 18 to 24 inch ...............

25 Almond, Pink, 12 to 18 inch ............. 7c

Almond, White, 2 to 3 feet ...............

35 Almond, White, 18 to 24 inches .........10 c

40 Almond, White, 12 to 18 inches .......... 7c

10 Althea, Purple, 2 to 3 feet ............. 5c

150 Althea, Red, 2 to 3 feet .................

100 Althea, Red, 18 to 24 inches .............. 40

125 Althea, White, 2 to 3 feet .............. 5c

15 Althea, White, 18 to 24 inches ..........4

300 Hydrangea, 2 to 3 feet ..................10

50 Hydrangea, 18 to 24 inches $\ldots . \ldots . . . .8 \mathrm{c}$

10 Japan, Quince, 2 to 3 feet ............ 8c

35 Japan Quince, 18 to 24 inches ..........6c

45 Japan Quince. 12 to 18 inches ............ 3c

10 Lilac, Purple, 18 to 24 inches ............ Sc

25 Lilac, White, 18 to 24 inches .............10c

25 Lilac, White, 12 to 18 inches $\ldots \ldots \ldots \ldots \ldots 6 \mathrm{c}$

20 Prunus Triloba, 2 to 3 feet ................ $10 \mathrm{c}$

25 Prunus Triloba, 18 to 24 inches ............ sc

10 Prunus Triloba, 12 to 18 inches ...........5

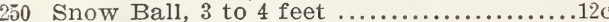

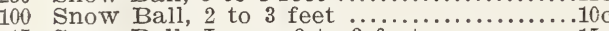

15 Snow Ball, Japan, 2 to 3 feet ................15c

300 Spirea, Van Houtte, 2 to 3 feet ............... 6 c

100 Spirea, Van Houtte, 12 to 18 inches....... 2c

\section{VINES.}

Each

200 Amelopsis Quinquefolia, No. 1 ..........6

175 Ampelopsis Veitchii, No. 1 ...................

50 Clematis, Jackmanii, 2 year, $\mathrm{No} .1 \ldots \ldots \ldots . .16 \mathrm{c}$

50 Clematis, Henryii, 2 year, No. 1 ............16c

100 Honeysuckle, Halls Japan, No. $1 . \ldots . . .68 \mathrm{c}$

100 Honeysuckle, Halls Japan, No. $1 \ldots . . . . .8 \mathrm{sc}$

75 Honeysuckle, Yellow Trumpet, No. 1.... 8c

30 Matrimony Vine, No. 1 ................ 5c

20 Wisteria Purple, No. 1 ................ sc

\section{PLANTS AND BULBS.}

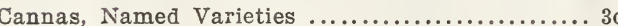

Each

Cannas, Mixed .............................. 2c

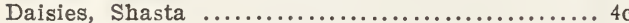

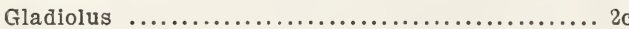

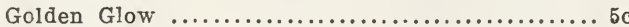

German Iris, Named Varieties ................ $3 \mathrm{c}$

German Iris, Mixed ......................... 2c

Hardy Salvia ...............................25c

Mexican Tube Roses ........................ 1c

Paeonias, rink ............................. $7 \mathrm{c}$

Paeonias, Red ............................... 8c

Paeonias, White ............................10

Sweet Williams ............................... $3 c$

ROSES.

Each

Climbing Roses, 2 year, No. 1 ...............

75 Dorothy Perkins

75 Queen of Prairie

Hybrid Perpetual Roses, 2 years, No. 1.........11c

30 Alfred Colombe

200 Anne d' Diesbach

20 Clo. Soupert

100 Coq. des Alps

50 Fisher Holmes

200 Frau Karl Druschkj

300 Gen. Jacqueminot

\section{Gruss au Teplitz \\ 20 Mad. Chas Wood \\ 100 Mad. Gabriel Luizet \\ 50 Magna Charta \\ 100 M. P. Wilder \\ 300 Paul Neyron \\ 75 Ulrich Bruner}

Each

Moss Roses, 2 year, No. 1

20 White Moss
Rambler Roses, 2 year, No. 1

Each

50 Blue (Veilchenblau)

204 Crimson

50 Philadelphia

25 White

10: Yellow

Each

Tea Roses, 2 year, No. 1

25 Mamon Cochet

10 Catherine Mermet

40 Etoile de Lyon

15 Francis Kruger

50 Helen Gould

50 K. A. Victoria

50 Pink Killarney

10 R. M. Henrietta

Miscellaneous Roses, 2 year No. 1

100 American Beauty .........................15c

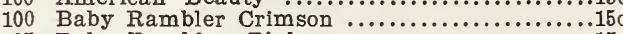

25 Baby Rambler, Pink $\ldots \ldots \ldots \ldots \ldots \ldots \ldots \ldots \ldots \ldots . .15 \mathrm{c}$

50 Baby Rambler, White $\ldots . \ldots \ldots \ldots \ldots \ldots \ldots \ldots . .15 \mathrm{c}$

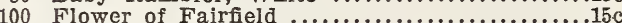

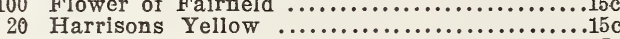

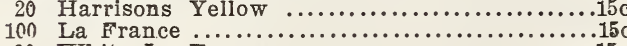

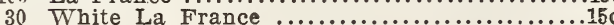

TREE ROSES.

5 Pink, No. 1

Each

P.nk, No............................35

15 Red, No. 1 ...............................35c

5 White, No. 1 ................................ $35 c$

5 Yellow, No. 1 ................................

\section{FOREST SFEDLINGS.}

Nursery Grown.

Per M.

2500 Box Elder, 3 to 4 feet $\ldots \ldots \ldots \ldots \ldots \ldots \ldots \ldots \ldots \ldots \ldots$

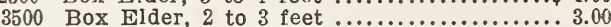

5000 Box Elder, 6 to 12 inches ................. 1.00

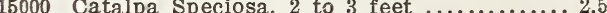

20000 Catalpa Speciosa, 18 to 24 inches .........2.2.25

12000 Catalpa Speciosa, 12 to 18 inches............ 1.75

8000 Catalpa Speciosa, 6 to 12 inches ............ 1.00

6500 Locust, Black, 3 to 4 feet .................4.00

15000 Locust, Black, 2 to 3 feet ................. 3.00

15000 Locust, Black, 18 to 24 inches ...........2.50

3000 Locust, Honey, 2 to 3 feet ................ 4.00

4000 Locust, Honey, 6 to 12 inches ............... 1.25

1000 Mulberry Russian, $z$ to 3 feet ................ 3.50

25000 Mulberry Russian, 18 to 24 inches ........ 2.50

25000 Mulberry Russian, 12 to 18 inches........ 1.50

50000 Mulberry Russian, 6 to 12 inches.......... 75

1500 Olives, Russian, 18 to 24 inches.............. 12.50

2000 Olives. Russian, 12 to 18 inches............ 10.03

1000 Olives Russian, 6 to 12 inches ........ 6.00

\section{HEDGF,}

Per M.

15000 Osage Orange, No. I .................. \$ 1.00

10000
8000 Osage Orange, No. $2 \ldots \ldots \ldots \ldots \ldots \ldots \ldots \ldots \ldots \ldots \ldots$
Osage Orange, No. 3

2000 Privet, California, 18 to 24 inches.......... 20.00

3000 Privet, California, 12 to 18 inches.......... 15.00

5000 Privet, California, 6 to 12 inches......... 6.00

625 Privet, Regel's, 18 to 24 inches........... 30.00

300 Privet, Regel's, 12 to 18 inches ......... 20.01

170 Privet, Regel's, 6 to 12 inches............ 10.00

Osage Orange can be furnished with tops on if desired.

\section{APPLE SEEDLINGS.}

Per M.

10000 No. 1 Straight, $3-16$ inch and all up....\$4.00 30000 No. 1 Branched, $1 / 4$ inch and all up .... 4.50 30000 No. 1 Branched, 3-16 inch and all up.... 3.50 10000 No. 3 For Lining out, about $2-16$ inch... 1.00

\section{SUPPLIES.}

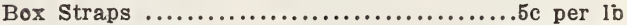

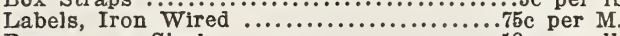
Rope, pure Sisal ......................... per lb Shingle Tow ....................\$1.00 per $100 \mathrm{lbs}$ 